\title{
Comparative studies on the success level of sonographic guided epidural steroid injection and its effectiveness with fluoroscopy techniques in treating lumbar radicular pain
}

\author{
Alireza Gelinimoghaddam, Masoud Hashemi*, Payman Dadkhah and Mehrdad Taheri \\ Department of Anesthesiology and Pain, Shahid Beheshti University of Medical Sciences, Tehran, Iran
}

\begin{abstract}
Limitations of using fluoroscopic guidance in epidural steroid injection have encouraged the researchers to look for an appropriate alternative method. Compared to fluoroscopy, sonography has exceptional properties that have made it a useful method. The present research seeks to study the success of sonographic guided epidural steroid injection and compare its effectiveness with fluoroscopy in treating lumbar radicular pain.This is a single-blind, randomized research conducted on 50 patients complaining about lumbar radicular pain. The participants were then randomly divided into two therapeutic groups consisting of 25 people: sonographic guided and fluoroscopy guided groups. The initial examination recorded the demographic information of each patient in his profile. The pain scale and performance disability and therapeutic satisfaction of each patient were measured 48 hours and 2 weeks after intervention and analyzed using statistical tests. The patients studied aged from 23 to 70 years (with an average of $45.74 \pm 16.02$ years) and were divided equally between the two groups in terms of gender. There was no statistically significant difference between the two groups in terms of contrast matter distribution, but the average injection procedure in sonography group $(101.22 \pm 36.48 \mathrm{~s})$ was significantly faster than what was observed in fluoroscopy group (311.46 $\pm 52.13 \mathrm{~s})(\mathrm{P}$-value $<0.05)$. There was no statistically significant difference between the two groups in terms of pain variable, performance disability and patients' satisfaction (P-value $>0.05$ ). There was no statistically significant correlation between gender and BMI with pain and performance disability in any one of these groups.The results have shown that sonographic and fluoroscopic tools have equal capabilities in epidural steroid injection. The superiority of sonography to fluoroscopy have made it possible to use this technique for epidural steroid injection.
\end{abstract}

KEY WORDS: SONOGRAPHY, FLUOROSCOPY, EPIDURAL, STEROID, LUMBAR RADICULAR PAIN

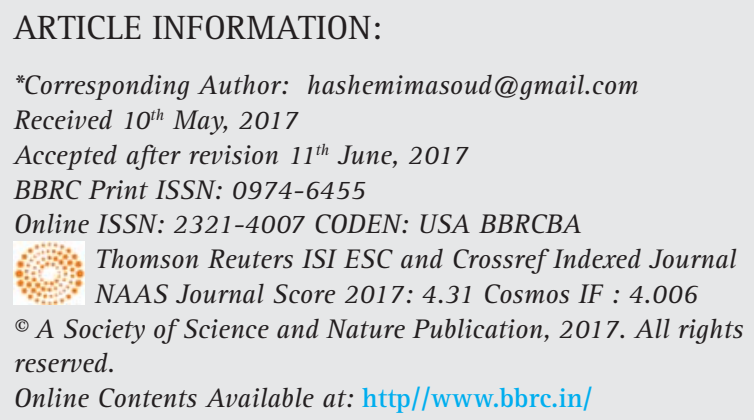




\section{INTRODUCTION}

Backache is the most common cause of limitations among people above the age of 45 in modern societies (Kozinoga et al., 2015). Backache is usually observed among the middle-aged people with women being more prone to it. The prevalence of backache among the elderly is reported to be $84 \%$ (Balague et al., 2012). Radicular pains are usually the result of nerve root inflammation or, sometimes, take place without mechanical stimulation (Chang et al., 2017). One of the major complications caused by intervertebral disc herniation is the epidural space and nerve roots inflammation. As clinical studies indicate, radicular pain caused by nerve root inflammation in epidural space is the result of disk leakage, pressure on nerve root arteries or stimulation of dorsal root ganglia due to spinal stenosis (Chang et al., 2017).

As radicular backache may be caused by epidural space and nerve root inflammation, the mechanism of inflammation suppression and repression by steroid medicines is largely used to treat this complication (Di Donato et al., 2010). Steroids are powerful anti-inflammatory medicines that reduce the inflammation caused in nerve roots by disk herniation or other pathologies in epidural space. Various studies conducted in this field have confirmed the effectiveness of epidural steroid injection in reducing the acute inflammation of nerve roots. In comparison to oral steroids, the superiority of epidural steroid injection lies in using lower amounts of medicines limited to the areas of the damaged nerve roots and reduced possibility of these medicines' side effects (Nampiaparampil et al., 2012). Radicular pain and backache relief, better cooperation of patient in physiotherapy, improved life quality, less consumption of analgesics and improved performance of the patient are some advantages of lumbar epidural steroid injection (Kurgansky et al., 2016). Although steroid epidural injections are largely used to reduce and control radicular pain, we should never forget the complication of this method including spinal cord infarction, hypertrophy of fat, menstrual changes and adrenal suppression (Bagheri et al., 2015, Brinks et al., 2010 Sehmbi et al., 2017).

Epidural steroid injection is a lumbar disk closed surgery method with various cognitive and therapeutic advantages. In cognitive terms, analgesia following local anesthetics injection to epidural space and the site of possible damage highlights the role of epidural space as the potential source of pain. Furthermore, the long term pain relief of the patient for several weeks following injection indicates existence of an inflammatory component in patient's pain and highlights the role of injection in reduction of this inflammatory procedure (White et al., 1980).
Fluoroscopy guided lumbar disk closed surgery is the method currently used for this complication. Studies have shown that injection is carried out in the wrong are in 30\% of the cases with no fluoroscopy and contrast confirmation (Manchikanti et al., 2004). High resolution of linear probe, harmonic imaging of tissue, two dimensional matrix probe technology, imaging with a wider viewing area, and duppler sonography power are some advantages of sonography in comparison with fluoroscopy. These advantages have turned ultrasound into a superior tool for analyzing skeletal-muscular disease. Previous studies have described sonography-guided caudal epidural steroid injection with an accuracy equal to fluoroscopy and with advantages such as the simplicity of this technique and refraining from being exposed to radiation (Park et al., 2013).

Current available researches conducted in this field are focused on measuring the correct placement of needle in caudal epidural space with no evidences presented concerning sonography-guided epidural injection and it has never been compared with fluoroscopy. Considering the constraints and limitations of fluoroscopy such as ionizing radiation and reduction of injection side effects such as random intravenous injection, researchers are looking for a better alternative for fluoroscopy. High resolution of probe, harmonic imaging of tissue, two dimensional matrix probe technology, imaging with a wider viewing area, higher pace, and the greater ease of injection are some advantages of sonography in comparison with fluoroscopy. These advantages have turned ultrasound into a superior tool for analyzing skeletalmuscular disease. Considering the potential of ultrasound technique, the present research seeks to study the success level of sonography-guided epidural steroid injection and compare its effectiveness with fluoroscopy in treating lumbar radicular pain through a single-blind, randomized, clinical trial.

\section{MATERIAL AND METHODS}

This is a clinical trial research. In a period of three months, as many as 50 patients aging 23 to 70 suffering from lumbar radicular pain whose diagnosis was confirmed through clinical files, medical examination results and electromyographic tests or were diagnosed with disk herniation or spinal stenosis in CTScan or MRI took part in the research. With due observation of medical ethics principles and after obtaining the consent of patients and making them aware of the research process, the participants took part in the research voluntarily. Systematic inflammatory diseases, treatment using anticoagulants, uncontrolled diabetes, history of allergic reaction to contrast material, diagnosed infection, dermal defects in the injected area, mental problems, history 
of injection or surgery within the last three months, need for non-steroid analgesics were exclusion criteria. The initial examinations recorded the demographic information of patients such their age, gender, height, and BMI. Visual analogue scale (VAS) and Oswestry disability index (ODI) questionnaires were completed by each patient. The patients were randomly and consecutively divided into two groups of sonography and fluoroscopy each consisting of 25 people. All patients assumed a prone position and the area was disinfected using alcohol solution. Dye contrast material was injected through sonography or fluoroscopy guide and after confirming standard contrast distribution pattern, 8 milliliter containing $80 \mathrm{mg}$ triamcinolone (40 $\mathrm{mg}$ if the patient had diabetes), 2 milliliter of 0.5\% Marcaine and up to 8 milliliter sterilized N.S solution was injected to the patient.

Sonography group: after anesthetizing the injection area, No. 18 Tuohy epidural needle was inserted through sonographic guide and scanning in parasagittal oblique view in accordance with in plane principles. Immediately after insertion of the needle into Ligamentum flavum, an auto-detect syringe was attached to the needle and inserted to the epidural space through LOR technique. To assure the correct placement in the post-injection are of 1-2 mm of contrast material, some images were captured in the lateral view. Having confirmed the standard distribution pattern, medicines with the above-mentioned combinations were injected in the area.

In fluoroscopy group: images were captured of the standard AP and lateral views. LOR technique was utilized to get to epidural space and paint using the contrast material. Having confirmed the standard distribution pattern, medicine with the above-mentioned mixture was injected in the area.

The present research studied the pattern of contrast distribution in terms of filling epidural space with contrast material. The length of injection time since the first imaging until the end of injection period was measured and recorded using a chronometer. The complications observed during the intervention such as vasovagal intervention, flashing or sudden lumbar pains within a few minutes following the injection were recorded in patients' profile. All injections and interventions in sonography group were conducted by a professional using $\mathrm{x}$-porte Sonosite sonography machine, c35xp high-frequency curve probe and Episure autodetect LOR syringe. In fluoroscopy group, ECORAY C-arm fluoroscopy X-ray systems was used to undertake epidural procedure. All patients were examined within 48 hours (T-1) and two weeks (T-2) following intervention in terms of fever, headache, hematoma, diabetes, high blood pressure, and passing pain. VAS was the most widely used pain measurement tool and ODI was set as the golden standard to assess the degree of disability and estimate the life quality of the patient suffering from backache. Pain levels of each patient were measured prior to procedure (T-0) and within 48 hours (T-1) and 2 weeks (T-2) after intervention using VAS and ODI scales and the measurements were analyzed using Mann Whitney U statistical test. To study patients' level of satisfaction within 48 hours (T-1) and 2 weeks (T-2) following the intervention, a questionnaire containing a five-option scale was utilized where 1 indicated dissatisfaction and 5 showed maximum satisfaction.

VAS: this scale consists of a $100-\mathrm{mm}$ flat horizontal line with one end titled "no pain" and the other side titled "the worst pain possible". The patients mark the pain scale they often feel on a 100-mm continuum of this flat line.

ODI questionnaire: this questionnaire includes 10 topics concerning pain levels, lifting objects, ability to take care of oneself, ability to walk, sexual performance, ability to stand, social life, sleep quality, and ability to go on a trip and each topic consists of 6 alternatives. The patients selected the option that most resembled their status (0 indicating very limited disability and 5 indicating highest level of disability) and the total scores were added up and multiplied by two (ranging from 0 to 100) where 0 indicated the lowest level of ability and 100 showed maximum ability. To study satisfaction level, a five-scale questionnaire was used with 1 indicating dissatisfaction and 5 showing maximum satisfaction. To make the research blind and prevent possible bias, radiologic images were simultaneously examined and studied by radiologists out of research and another physician not aware of injection method.

The resulting raw data was analyzed using IBM SPSS statistics v.23 and Kolmogorov-Smirnov Test was used to study the normality of all data and proper statistical tests were used in accordance with the normality status. Chi-square, Mann Whitney U and Spearman correlation tests were used to analyze the data. The level of statistical significance (P-value) in this research was set to less than 0.05 .

\section{RESULTS AND DISCUSSION}

As many as 50 patients suffering from lumbar radicular pain participated in this research and were randomly divided into sonography-guided and fluoroscopy-guided groups each consisting of 25 people. The patients' age ranged from 23 to 70 years old $(45.74 \pm 16.02$ years) and they were divided randomly in terms of their age in both groups (Figure 1). Other demographic information is presented in table 1 .

Contrast distribution pattern of both groups was studied in terms of filling epidural space using contrast material and the length of injection procedure and 


\begin{tabular}{|l|l|l|l|l|}
\hline \multicolumn{6}{|l|}{ Table 1. Demographic information of the patients participating in the research } \\
\hline Variables & Minimum & Maximum & Mean & SD \\
\hline Age (year) & 23.00 & 70.00 & 45.74 & 16.02 \\
\hline Height $(\mathrm{cm})$ & 140.00 & 188.00 & 161.26 & 12.60 \\
\hline Weight $(\mathrm{kg})$ & 45.00 & 92.00 & 69.88 & 12.73 \\
\hline BMI & 18.73 & 36.73 & 26.84 & 3.78 \\
\hline
\end{tabular}

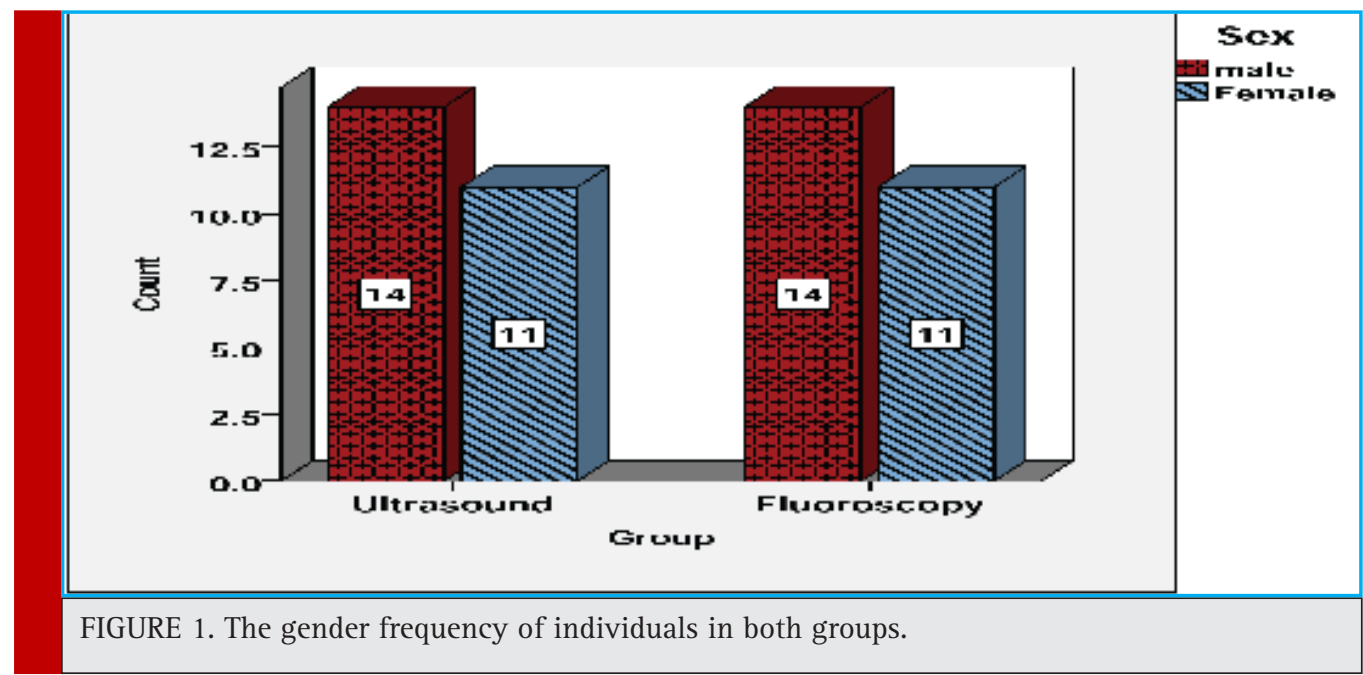

the resulting data was recorded. Chi-square and Mann Whitney U tests were used to analyze contrast distribution pattern and injection procedure length respectively. The results showed contrast distribution pattern in both groups was completely similar. In both groups, there were 24 standard compatible contrast distribution pat- terns and only 1 non-compatible pattern (Figure 2). As a result, there was no statistically significant difference between the two groups in terms of contrast distribution pattern (P-value $>0.05$ ). The average lengths of injection procedure in both groups were completely different. The injection procedure in sonography group had an

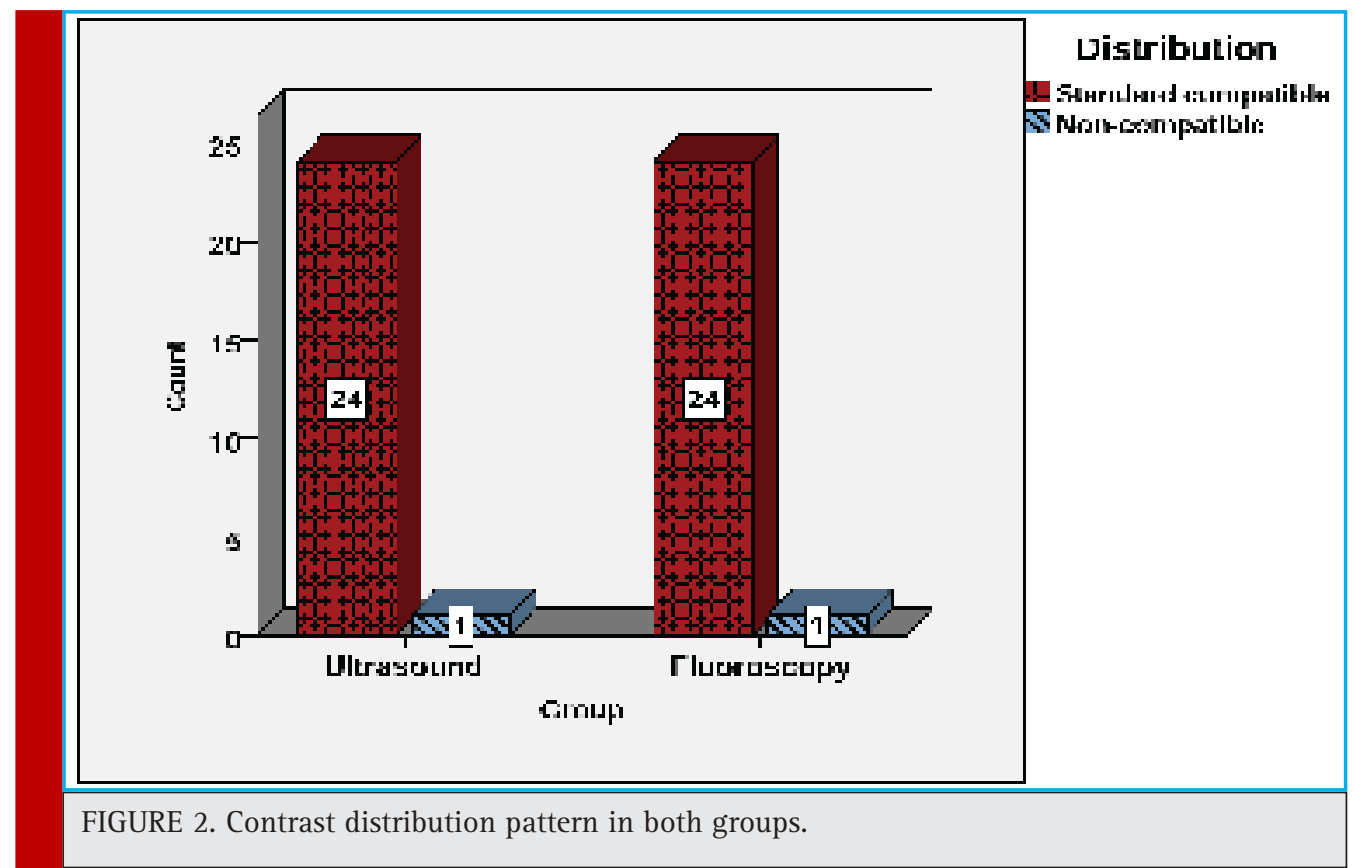


Table 2. Statistical details of contrast distribution pattern and the length of injection procedure in groups studied

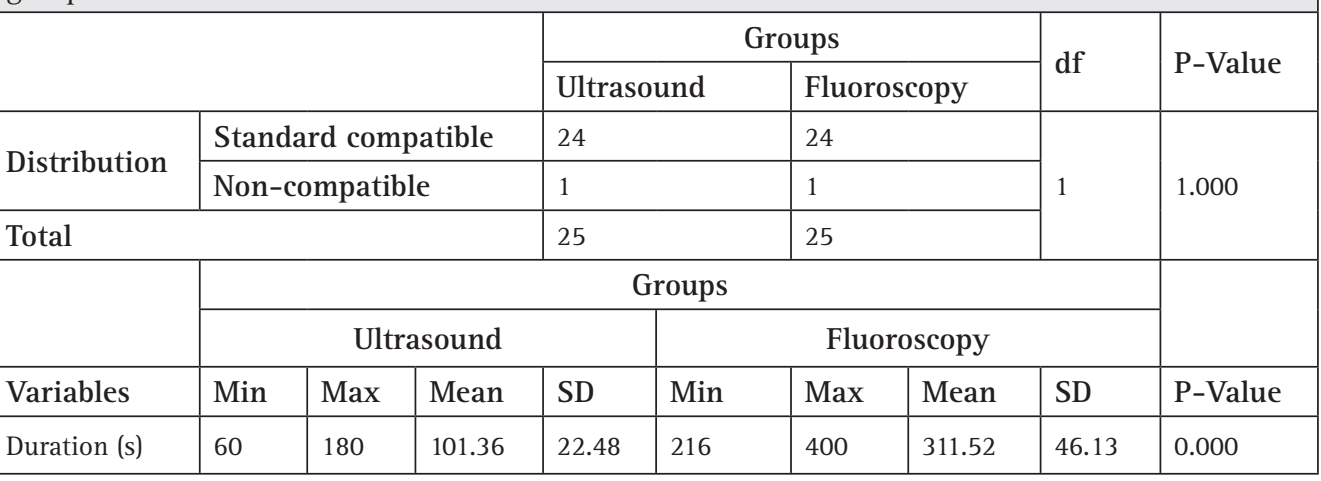

average length of $101.22 \pm 36.48 \mathrm{~s}$, while this procedure in the other group lasted $311.46 \pm 52.13 \mathrm{~s}$ (P-value < 0.05) (Table 2).

The information collected from the participating groups concerning VAS and ODI prior to procedure (T-0) and within 48 hours (T-1) and 2 weeks (T-2) after intervention and patients' satisfaction levels in both groups within 48 hours (T-1) and two weeks (T-2) after intervention were collected using a questionnaire with a 5-alternative scale and analyzed using Mann Whitney $U$ test. The results of these statistical tests failed to find any statistically significant difference between the two groups in terms of these variables (P-value $>0.05$ ). The performance of sonography and fluoroscopy methods had no significant influence on VAS and ODI and patients' satisfaction (Tables 3 \&t 4).

The present research also studied the effect of age, gender and BMI on VAS and ODI within 48 hours (T-1) and two weeks (T-2) following epidural steroid injection under sonography and fluoroscopy guidance.
The results found a statistically significant correlation between age and VAS and ODI within two weeks after intervention (ODI_2 \& VAS_2) in sonography group and ODI_2 in fluoroscopy group (P-value $<0.05)$. As the age grew older and older, the level of these variables also increased (in other words, the post-procedure pain reduction and performance power of the patients would increase in older ages). There was no statistically significant correlation between sex and BMI with VAS and ODI within 48 hours (T-1) and two weeks (T-2) after epidural steroid injection (P-value $>0.05$ ) (Table 5).

Spinal disk bulging and degenerative narrowing of spinal canal are two major causes of radicular lumbar pains (Cyteval et al., 2006). Laboratory documents have pointed to inflammation of nerve roots as the mechanism of causing pain (Ghahreman et al., 2010). Experts have recommended injection of steroids in epidural space in order to reduce pain and inflammation. Fluoroscopy guidance is the method used for injection in epidural space which has countless limitations and dangers. Considering the high

Table 3. A comparison of VAS and ODI before the procedure (T-0), within 48 hours (T-1) and two weeks (T-2) following intervention in both groups

\begin{tabular}{|l|l|l|l|l|l|l|l|l|l|}
\hline Variables & Min & Max & Mean & SD & Min & Max & Mean & SD & P-Value \\
\hline VAS_0 & 4 & 10 & 7.52 & 1.38 & 6 & 9 & 7.20 & 0.86 & 0.248 \\
\hline ODI_0 & 42 & 88 & 67.28 & 12.48 & 50 & 88 & 71.84 & 9.62 & 0.167 \\
\hline VAS_1 & 2 & 8 & 3.88 & 1.26 & 2 & 8 & 3.76 & 1.12 & 0.544 \\
\hline ODI_1 & 20 & 80 & 37.92 & 12.07 & 26 & 82 & 40.56 & 10.97 & 0.220 \\
\hline VAS_2 & 2 & 8 & 3.12 & 1.36 & 2 & 8 & 3.00 & 1.19 & 0.827 \\
\hline ODI_2 & 20 & 80 & 30 & 12.27 & 20 & 80 & 32.48 & 11.52 & 0.187 \\
\hline
\end{tabular}

Table 4. Comparing levels of patients' satisfaction within 48 hours (T-1) and two weeks (T-2) following intervention in both groups

\begin{tabular}{|l|l|l|l|l|l|l|l|l|l|}
\hline Variables & Min & Max & Mean & SD & Min & Max & Mean & SD & P-Value \\
\hline Satisfaction-1 & 1 & 5 & 4.48 & 0.91 & 1 & 5 & 4.56 & 0.86 & 0.717 \\
\hline Satisfaction-2 & 1 & 5 & 4.52 & 0.962 & 1 & 5 & 4.64 & 0.86 & 0.667 \\
\hline
\end{tabular}




\begin{tabular}{|c|c|c|c|c|c|c|}
\hline \multirow{2}{*}{ Groups } & \multirow{2}{*}{ Variables } & \multirow{2}{*}{ Tests } & \multicolumn{4}{|c|}{ P-Value } \\
\hline & & & VAS_1 & ODI_1 & VAS_2 & ODI_2 \\
\hline \multirow{3}{*}{ Ultrasound } & Age & Spearman & 0.202 & 0.113 & 0.011 & 0.044 \\
\hline & BMI & Spearman & 0.511 & 0.741 & 0.992 & 0.834 \\
\hline & Sex & Mann Whitney & 0.586 & 0.093 & 1.000 & 0.473 \\
\hline \multirow{3}{*}{ Fluoroscopy } & Age & Spearman & 0.245 & 0.892 & 0.129 & 0.018 \\
\hline & BMI & Spearman & 0.236 & 0.868 & 0.157 & 0.063 \\
\hline & Sex & Mann Whitney & 0.722 & 0.283 & 0.397 & 0.320 \\
\hline
\end{tabular}

potential and unique properties of ultrasound method compared with fluoroscopy, this tool can be considered a good alternative for fluoroscopy. The present research sought to compare sonography- and fluoroscopy-guided epidural steroid injection in radicular lumbar pain. Although no similar researches were found in our review of literature, there are similar and comparable researches in this field whose results can be compared with those of the current study. A research was conducted on efficiency of caudal epidural injection guided by ultrasound by Nikooseresht et al in 2014.

They studied as many as 240 patients suffering from sciatica backache. Epidurogram confirmed the success of 230 cases out of 240 injections (95.8\%). They arrived at the conclusion that ultrasound is a safe, quick and reliable method to assess various variations of Sacral hiatus and, also, epidural injection (Nikooseresht et al., 2014). Yoon et al (2005) studied the effectiveness of ultrasound duppler and fluoroscopy in finding intravascular flow in order to confirm placement of needle in epidural caudal space among 53 patients suffering from backache and sciatica. The medicine was injected successfully into epidural caudal space through sonography in 52 people. They concluded that sonography was a reliable imaging method for caudal epidural steroid injection. What's more, its advantages such as the ease and no risk of ionization radiation turn it to a more appropriate choice than fluoroscopy (Yoon et al., 2005). Another research conducted by Chen et al studied the correct performance of ultrasound in locating sacral hiatus and guiding the needle to caudal epidural space in the kidneys of 70 patients. Sacral hiatus was located carefully using ultrasound in all patients and epidural caudal needle was successfully guided to the epidural space. The accuracy of needle placement in epidural space as directed by ultrasound was confirmed by injection of contrast material and fluoroscopy (Chen et al., 2004).

The results of the researches conducted by Nikooseresht, Yoon and Chen are completely in line with those achieved in the present study. The only difference between these studies and those of our research lies in the site of injection. Our study was based on a new approach and investigated sonography-guided steroid injection procedure to the epidural space while they carried out this research in epidural caudal space. Obernauer et al studied factors such as accuracy, time, radiation level, safety and pain reduction in ultrasound- and CT scan-guided pre-radicular injection and compared them against one another. The results of this research conducted on 40 patients confirmed the 100\% accuracy of ultrasound-guided intervention. The average length of needle placement in sonography was 2:21 minutes, while this length in CT Scan-guided method was 10:33 minutes. Both groups showed a similar reduction in pain levels and no difference was observed between the techniques in such terms. They finally arrived at the conclusion that ultrasound-guided pre-radicular injection has an equal therapeutic accuracy and efficiency than CT scan guidance. This procedure requires a shorter time and has no risk of ionization ray (Obernauer et al., 2013).

Although this procedure is completely different from our procedure, the results they have achieved (no difference in therapeutic efficiency of methods and the high speed of procedure in ultrasound method) are in line with our research.

\section{CONCLUSION}

With reference to the results of the present research, we may conclude that there is no difference between the ability of sonography and fluoroscopy in steroid epidural injection. Considering the special advantages of sonography such as less radiation than fluoroscopy, it is concluded that sonography can be used in steroid epidural injection procedure to treat radicular pains, just like fluoroscopy.

\section{REFERENCES}

Bagheri S.M., Gharib M.H. (2015) How can a radiologist reveal more practical information using dynamic study of cavern- 
osal artery after injection of vasoactive agents? Iran J Radiol. 12(1):e12556.

Balague F., Mannion A.F., Pellise F., Cedraschi C. (2012) Non-specific low back pain. Lancet (London, England). 379(9814):482-91.

Brinks A., Koes B.W., Volkers A.C., Verhaar J.A., Bierma-Zeinstra S.M. (2010) Adverse effects of extra-articular corticosteroid injections: a systematic review. BMC Musculoskeletal Disorders. 11(1):206.

Chang M.C., Cho Y.W., Ahn S.H. (2017) Comparison between bipolar pulsed radiofrequency and monopolar pulsed radiofrequency in chronic lumbosacral radicular pain: A randomized controlled trial. Medicine (Baltimore). 96(9):e6236.

Chen C.P., Tang S.F., Hsu T-C., Tsai W-C., Liu H-P., Chen M.J., et al. (2004) Ultrasound guidance in caudal epidural needle placement. The Journal of the American Society of Anesthesiologists. 101(1):181-4.

Cyteval C., Fescquet N., Thomas E., Decoux E., Blotman F., Taourel P. (2006) Predictive factors of efficacy of periradicular corticosteroid injections for lumbar radiculopathy. American journal of neuroradiology. 27(5):978-82.

Di Donato A., Fontana C., Alemanno D., Di Giacomo A. (2010) Epiduroscopy in treatment of degenerative chronic low back pain: A prospective analysis and follow-up at 60 months. Clinical Research and Regulatory Affairs. 27(3):69-74.

Ghahreman A., Ferch R., Bogduk N. (2010) The efficacy of transforaminal injection of steroids for the treatment of lumbar radicular pain. Pain Medicine. 11(8):1149-68.

Kozinoga M., Majchrzycki M., Piotrowska S. (2015) Low back pain in women before and after menopause. Przeglad Menopauzalny = Menopause Review. 14(3):203-7.

Kurgansky K.E., Rodriguez S.T., Kralj M.S., Mooney J.J., Dinakar P., d'Hemecourt P.A., Hedequist D.J., Proctor M.R., Berde C.B. (2016) Epidural Steroid Injections for Radiculopathy and/or Back Pain in Children and Adolescents: A Retrospective
Cohort Study With a Prospective Follow-Up. Reg Anesth Pain Med. 41(1):86-92.

Manchikanti L, Cash KA, Pampati V, McManus CD, Damron KS.2004 Evaluation of fluoroscopically guided caudal epidural injections. Pain physician. 7(1):81-92.

Nampiaparampil D.E., Nampiaparampil G.M., Nampiaparampil R.G. (2012) Oral opioid analgesics vs. spinal steroid injections in the treatment of low back pain syndromes. American journal of physical medicine \&t rehabilitation. Am J Phys Med Rehabil. 91(2):162-76.

Nikooseresht M., Hashemi M., Mohajerani S.A., Shahandeh F., Agah M. (2014) Ultrasound as a screening tool for performing caudal epidural injections. Iranian Journal of Radiology. 11(2): e13262.

Obernauer J., Galiano K., Gruber H., Bale R., Obwegeser A.A., Schatzer R., et al. (2013) Ultrasound-guided versus computed tomography-controlled periradicular injections in the middle and lower cervical spine: a prospective randomized clinical trial. European Spine Journal. 22(11):2532-7.

Park Y., Lee J.H., Park K.D., Ahn J.K., Park J., Jee H. (2013) Ultrasound-guided vs. fluoroscopy-guided caudal epidural steroid injection for the treatment of unilateral lower lumbar radicular pain: a prospective, randomized, single-blind clinical study. American journal of physical medicine \&t rehabilitation. 92(7):575-86

Sehmbi H., D’Souza R., Bhatia A. (2017) Low Back Pain in Pregnancy: Investigations, Management, and Role of Neuraxial Analgesia and Anaesthesia: A Systematic Review. Gynecol Obstet Invest. doi: 10.1159/000471764. [E pub ahead of print]

White A.H., Derby R., (1980) Wynne G. Epidural injections for the diagnosis and treatment of low-back pain. Spine. 5(1): 78-86.

Yoon J.S., Sim K.H., Kim S.J., Kim W.S., Koh S.B., Kim B-J. (2005) The feasibility of color Doppler ultrasonography for caudal epidural steroid injection. Pain.118(1):210-4. 